CASE REPORT

\title{
Evolution of childhood central diabetes insipidus into panhypopituitarism with a large hypothalamic mass: is 'Iymphocytic infundibuloneurohypophysitis' in children a different entity?
}

\author{
Mohamad Maghnie, Eugenio Genovese ${ }^{1}$, Maria Grazia Sommaruga ${ }^{1}$, Maurizio A ricò, Davide Locatelli ${ }^{2}$, \\ Elena Arbustini ${ }^{3}$, Stefano Pezzotta ${ }^{2}$ and Francesca Severi \\ Departments of Pediatrics, ${ }^{1}$ Radiology, ${ }^{2}$ Neurosurgery and ${ }^{3}$ Histopathology, University of Pavia, IRCCS Policlinico S. M atteo, Pavia, Italy \\ (Correspondence should be addressed to M M aghnie, Department of Pediatrics, University of Pavia, IRCCS Policlinico S. M atteo, 27100 Pavia, Italy)
}

\begin{abstract}
We report on a 15 -year-old girl who had presented with acute onset central diabetes insipidus at the age of 8 years; this was followed by growth failure due to acquired growth hormone deficiency. Initial magnetic resonance imaging showed a uniformly enlarged pituitary stalk and absence of posterior pituitary hyperintensity. Frequent patient examination and magnetic resonance imaging gave unchanged results until after 5 years a large hypothalamic mass and panhypopituitarism were found. Dynamic magnetic resonance imaging documented hypothalamic - pituitary vasculopathy. Histopathological examination revealed perivascular inflammatory lymphoplasmic infiltrates with no granulomatosis or necrosis and negative staining for S-100 protein, suggesting autoimmuneinflammatory disease (lymphocytic infundibuloneurohypophysitis?). The response to glucocorticoid pulses (30 mg/kg per day for 3 days i.v.) was favorable, the hypothalamic mass being halved and partial anterior pituitary function recovery maintained for 2 years after the start of treatment. We suggest that long-term surveillance is needed for isolated and chronic thickening of the pituitary stalk and that dynamic magnetic resonance imaging can contribute to the demonstration of hypothalamic- pituitary vascular impairment associated with local vasculitis.
\end{abstract}

European Journal of Endocrinology $139635-640$

\section{Introduction}

Acquired central diabetes insipidus in childhood has a varied etiology including infiltrative mass (1), Langerhans cell histiocytosis ( $(\mathrm{CH})(2)$, and autoimmune and inflammatory diseases $(3-5)$. The idiopathic forms appear to be the most common (6-8). Thanks to magnetic resonance imaging (MRI), an early diagnosis of pituitary stalk infiltration with lack of posterior pituitary hyperintensity is now possible (2-5). In particular, the presence of an isolated thickened pituitary stalk is a frequent finding with evolution to either germinomas $(1,9)$ or a self-limiting disease as in lymphocytic infundibuloneurohypophysitis (3). Diagnosis of the cause of central diabetes insipidus remains a challenge in many cases.

\section{Case report}

In September 1990, an 8-year-old girl presented with a history of weight loss $(3 \mathrm{~kg})$, temporal headache, fatigue and polyuria-polydipsia. Past medical history including psychosocial disturbances, familial polyuria-polydipsia or kidney disease was unremarkable. Physical and ophthalmological examinations were normal. Her height was $130.7 \mathrm{~cm}$ and weight $27.4 \mathrm{~kg}$ (both at 50 75 Tanner centile). Erythrocyte sedimentation rate, Creactive protein, serum protein and immunoglobulins, blood cell count, liver and kidney functions were normal. Urinary volume was 7-7.5 litres/day with urine osmolality less than $100 \mathrm{mOsm} / \mathrm{kg}$. The diagnosis of central diabetes insipidus was made using a standard water deprivation test (10) and the child was started on nasal 1-desamin 0-8-D-arginine vasopressin at a dose of $5 \mu \mathrm{g}$ twice daily. Growth hormone (GH) secretion evaluated by insulin-induced hypoglycemia, arginine and oral L-dopa was impaired, with $\mathrm{GH}$ peaks of $0.6,1.0$ and $0.3 \mathrm{ng} / \mathrm{ml}$ respectively. Evaluation of the remaining anterior pituitary hormones including thyrotropin, adrenocorticotropin and follicle-stimulating hormone (FSH)/luteinizing hormone (LH) revealed normal thyroid-adrenal-gonad function; basal prolactin levels were normal. 
T1-weighted MRI of the hypothalamic-pituitary area showed a uniformly enlarged pituitary stalk and absence of posterior pituitary hyperintensity, while the anterior pituitary was normal (pituitary height $5 \mathrm{~mm}$, volume $310 \mathrm{~mm}^{3}$ ) (Fig. 1). Further diagnostic assessments including purified protein derivative tuberculin testing, antinuclear, thyroid and pituitary antibodies and complement activity were normal. Spontaneous nitroblue tetrazolium reduction assay performed as a marker of peripheral blood monocyte hyperactivation (11) showed $5 \%$ positivity (normal value $<10 \%$ ); $\alpha$-fetoprotein $(<5 \mathrm{ng} / \mathrm{ml})$ and the $\beta$-subunit $(<5 \mathrm{mU} / \mathrm{ml})$ of human choriogonadotropin were not found in either the plasma or the cerebrospinal fluid. Analysis of the cerebrospinal fluid gave normal results for cellular content, protein and immunoglobulins as well as for cytology. A chest $X$-ray failed to reveal enlarged lymph nodes and skull X-ray showed no abnormalities. Abdominal ultrasonography was negative and total body gallium scan revealed a hot spot on the maxillary sinus which was not confirmed by X-ray. Because of growth arrest (growth velocity $0.5 \mathrm{~cm} / 6$ months), $\mathrm{GH}$ treatment was started at theage of 8.5 yearsat a dose of $15 \mathrm{U} / \mathrm{m}^{2}$ per week in six doses s.c., leading to an increase in growth velocity of up to $9 \mathrm{~cm} /$ year. The patient was followed up at 6 month intervals for endocrine function and for MRI at 3 month intervals in the first 6 months and then every 10 months. There was no evidence of pituitary disease progression or MRI variation. Dynamic MRI performed 24 months after presentation revealed abnormal hypothalamic-pituitary vascularization suggested by the delayed initial increase in the posterior pituitary and markedly delayed increase in the anterior pituitary gland (12). In normal controls the temporal distribution of the contrast agent correlates closely with the vascular anatomy of the pituitary region. The contrast material flows first to the area of the inferior hypophyseal artery, then to that of the superior hypophyseal artery; the anterior pituitary tissue near the lower infundibular stem thus receives a dual supply. The normal pattern of en hancement of the pituitary gland after the injection of gadopentetate dimeglumine (Gd-DTPA) is represented first by enhancement of the posterior pituitary lobe, which occurs simultaneously with that of the straight sinus, followed by the anterior pituitary gland (12). The reason why this venous vessel is increased simultaneously

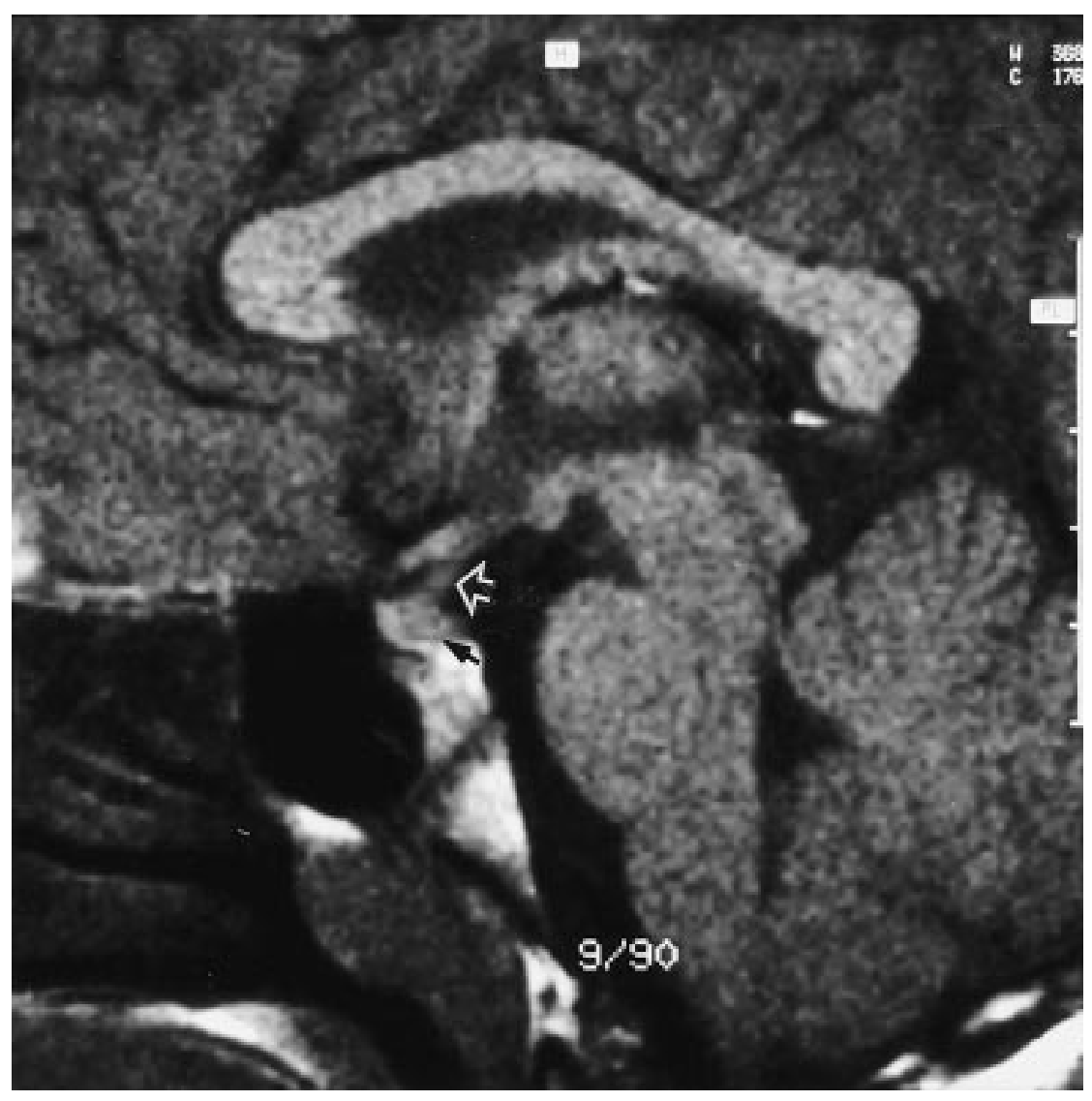

Figure 1 Sagittal T1-weighted MRI at presentation (September 1990) showing a uniformly thickened pituitary stalk (white open arrowhead). Posterior pituitary hyperintensity is absent (black arrowhead). 
with the posterior pituitary lobe may be that the blood supply flows faster in this large-caliber vessel than in a small perfusion system such as the inferior hypophyseal artery.

The start of puberty (Tanner II for breast) was first seen at the age of 10 years and progressed until Tanner III at the age of 11.6 years. At 56 months after presentation (8 months after the last examination), MRI showed a huge hypothalamic-pituitary mass, rapidly enhanced by Gd-DTPA with reduction of the anterior pituitary height $(2.4 \mathrm{~mm})$ and volume $\left(102 \mathrm{~mm}^{3}\right)$ (Fig. 2).

The patient was asymptomatic, and physical and ophthalmological examinations were normal. Thyroid (low free thyroxine with normal basal thyrotropin levels) and adrenal (low basal adrenocorticotropin and morning cortisol levels) dysfunction were documented. $\mathrm{LH}$ and $\mathrm{FSH}$ response to gonadotropin-releasing hormone were 'apparently' normal (16.5 and $9.8 \mathrm{mIU} / \mathrm{l}$, partial defect?). The pubertal stages were Tanner P4 and B3 while uterine length was $3.7 \mathrm{~cm}$ at pelvic ultrasound. A nalysis of cerebrospinal fluid gave normal results including cytology. Organ-specific antibodies against pituitary, arginine vasopressin cells, adrenal and thyroid were performed as previously described (13), and non-organ-specific antibodies including antineutrophil cytoplasmic antibodies were negative. At open hypothalamic-pituitary stalk biopsy, in August 1995, the tissue appeared very hard. Histopathological examination revealed perivascular inflammatory lymphoplasmacytic infiltrates with few granulocytes and macrophages, compatible with the usual findings of lymphocytic infundibuloneurohypophysitis or lymphocytic hypophysitis. Rosenthal fibers were identified but they were not suggestive of pilocytic astrocytoma, and staining for S-100 protein was negative. The patient was treated with high-dose prednisolone $(30 \mathrm{mg} / \mathrm{kg}$ per day, total dose of $2.4 \mathrm{~g}$ in 20 min infusion for 3 days) according to current LCH-I treatment protocol (14). MRI performed 1 month later showed a $50 \%$ decrease in the hypothalamic mass (Fig. 2) with delayed hypothalamicpituitary vascularization (Fig. 3). Thyroid, adrenal
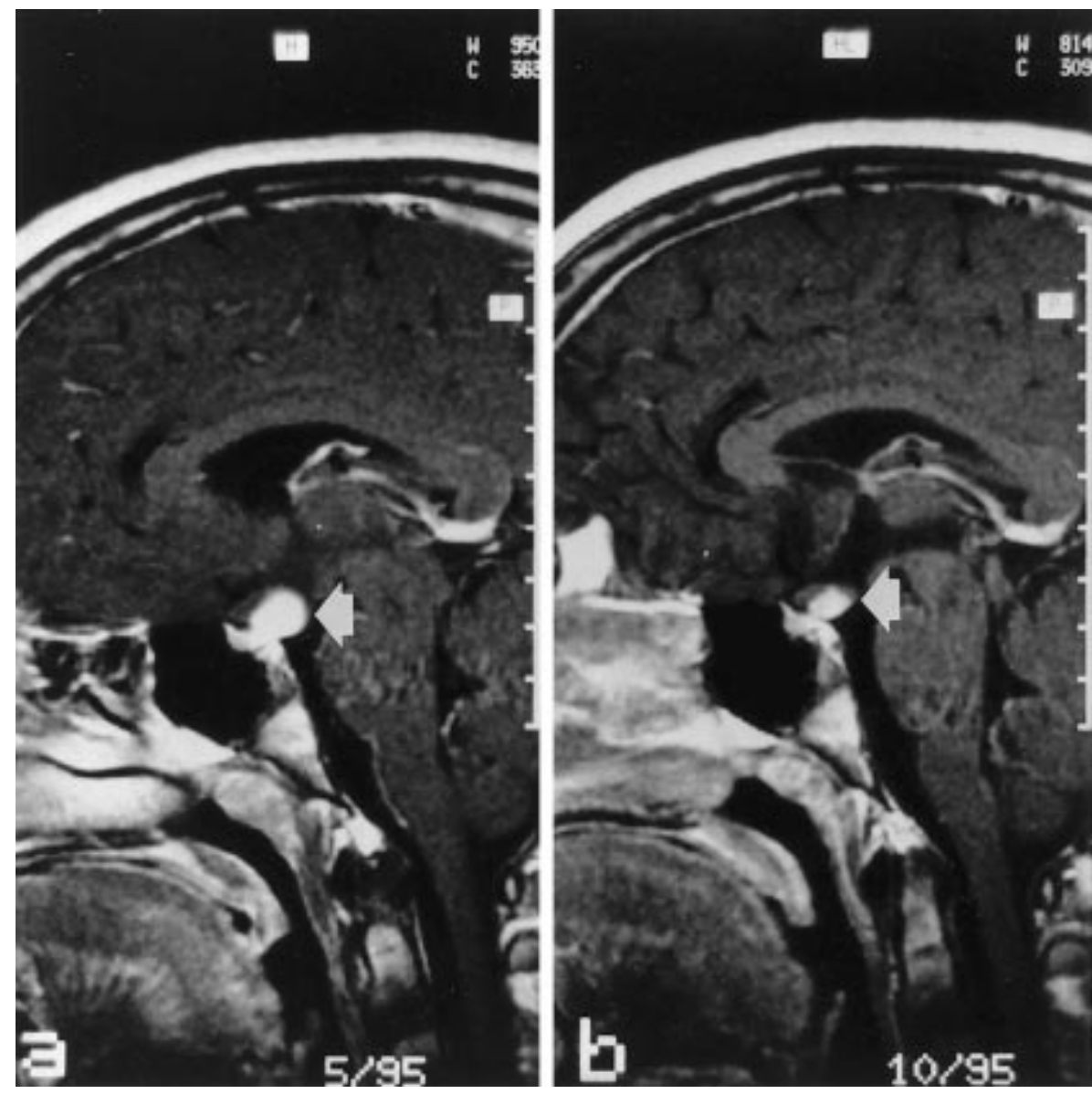

Figure 2 Sagittal T1-weighted MRI performed during the follow-up (May 1995) showing a large hypothalamic-pituitary mass (white arrowhead) homogeneously enhanced after Gd-DTPA administration (a). Reduction of the mass after steroid treatment (b). 


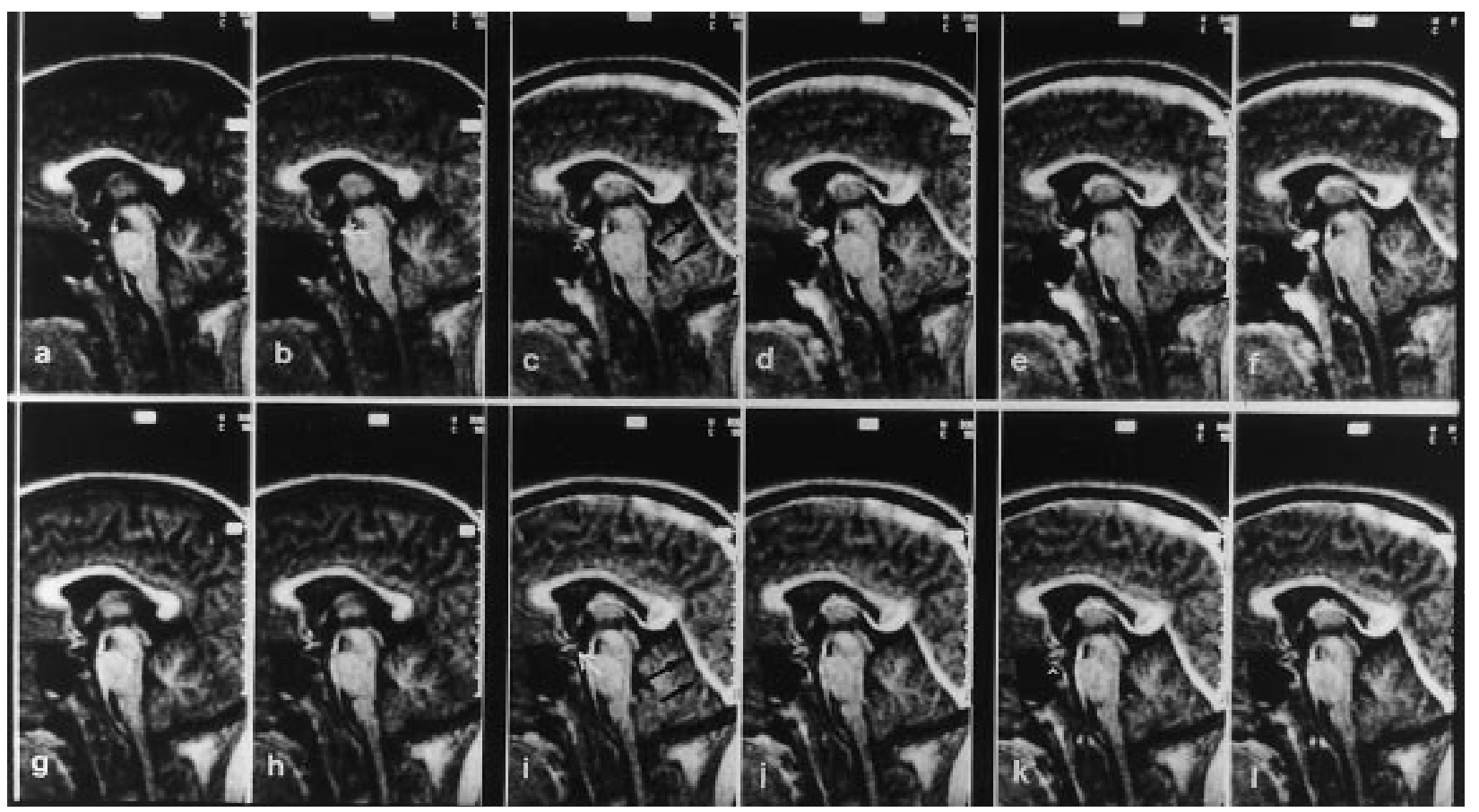

Figure 3 Sagittal dynamic MRI at the time of mass discovery (a-f) showing a very rapid increase (b) (white arrow) before the increase in the straight sinus seen one frame later (c) (double black arrows). The initial increase in the anterior pituitary is also visible (open white arrowhead) (c). Dynamic MRI after steroid treatment $(\mathrm{g}-\mathrm{l})$ shows an increase in the remaining mass (i) (white arrow) simultaneously with that of the straight sinus (i) (double black arrows). The initial increase in the anterior pituitary is very late (k) (open white arrowhead), indicating a vascular derangement pattern.

and gonadal (FSH and $\mathrm{LH}$ response to gonadotropinreleasing hormone) functions recovered but diabetes insipidus and hypopituitarism persisted. In June 1996 hypogonadotropic hypogonadism was diagnosed. At the last examination in September 1997, thyroid and adrenal function were still normal, and standard and dynamic MRI findings were unchanged.

\section{Discussion}

This girl presented with acute onset central diabetes insipidus and GH deficiency with thickened pituitary stalk, and unexpectedly progressed to panhypopituitarism with a large hypothalamic mass. The first MRI examination suggested an infiltrativeand/or inflammatory disease of the pituitary stalk (2) including granular cell tumor (15), juvenile pilocytic astrocytoma (16), germinomas $(1,9)$, meningiomas (17), LCH (4), tuberculosis and sarcoidosis (3).

The usual course of a thickened pituitary stalk is spontaneous regression or evolution into a regional tumor as the result of germinoma $(1,5)$. Germinomadependent growth is usually progressive, and, in most reported cases, overt mass is visible within the first 3-4 years (1). In our patient, close follow-up excluded progressive grow th but sudden bulging was visible 5 years later. At that time, when we decided to perform an open biopsy, the differential diagnosis included germinoma and other non-neoplastic conditions such as lymphocytic infundibuloneurohypophysitis (5), lymphocytic hypophysitis (18-20) and necrotizing infundibulohypophysitis (21). The inflammatory picture shown on histological examination did not suggest granulomatosis or necrosis and excluded LCH, germinoma and pilocytic astrocytoma. Given the age at presentation, the clinical course and the MRI pictures including anterior pituitary hypoplasia and abnormal hypothalamic-pituitary vascularization, this case is clearly different from the few reported of local inflammatory processes. Lymphocytic infundibuloneurohypophysitis was excluded because it has been reported in adults as being confined to the posterior pituitary and being self-limited (5). Lymphocytic hypophysitis as classically conceived was unlikely for several reasons: $90 \%$ of reported cases were in females but they were older except in one case (22) and in at least $65 \%$ associated with pregnancy; $60 \%$ had symptoms such as headache and visual defect; $40 \%$ had hyperprolactinemia with functional involvement confined to the anterior pituitary (19-21); the MRI picture was that of pituitary enlargement $(80 \%)$ mimicking pituitary adenoma (20). None of these features was found in our patient. The absence of necrosis and the long history of the disease are strikingly 
different from what is reported for so-called 'necrotizing infundibulohypophysitis': in the only report, two adult men had hypopituitarism and sellar mass, with a very short ( 1 and 3 weeks) history of diabetes insipidus (21). We believe that the conditions described, apparently independent, possibly belong to a unique spectrum of inflammatory conditions affecting the hypothalamicpituitary area. Inflammatory virus-mediated/autoimmune/vascular phenomena play a role in such a scenario but our current ability to identify markers of these pathogenic mechanisms clearly remains inadequate. This appears to be much more complicated in children, for whom criteria for diagnosis of lymphocytic infundibuloneurohypophysitis and lymphocytic hypophysitis are still lacking. This case shows the need to revise our classification schemes for diagnosis of lymphocytic infundibuloneurohypophysitis and lymphocytic hypophysitis presenting with diabetes insipidus, anterior pituitary deficiencies and thickened pituitary stalk during childhood and adolescence. We believe that in children lymphocytic infundibuloneurohypophysitis may be a different entity from that reported in adults and that anterior pituitary deficiencies may be more common than is usually thought. The involvement of the pituitary stalk, the posterior pituitary lobe and the anterior pituitary indicates that the term 'lymphocytic infundibulohypophysitis' is more appropriate than lymphocytic infundibuloneurohypophysitis. In the present case, as in that reported by Cemeroglu et al. (22), the possible role of puberty in disease worsening (are puberty and disease worsening criteria for lymphocytic hypophysitis?) remains an interesting subject of speculation.

The contribution of dynamic MRI to the demonstration of hypothalamic-pituitary vascular impairment may be significant in this case. Signs of vasculopathy were already associated with isolated thickened pituitary stalk (12), while rearrangement of the local blood supply was probably secondary to arterial dilatation or neovascularity. The local vascular changes in the course of inflammation may explain the early increase in the hypothalamic mass enhancement, which may be responsible for the greater blood perfusion and faster blood flow than in a large-caliber venous vessel such as the straight sinus. The rapid increase in the anterior pituitary could be secondary to the fact that the high perfused large mass invading the low infundibular stem and the upper pituitary gland provide a major blood supply to this area also. Dynamic MRI seems to be a promising technique for the differential diagnosis of mass lesion involving the hypothalamus and pituitary stalk, as the pattern of rapid increase shown in the presence of inflammatory mass is different from that recently observed in germinoma and craniopharyngioma (M Maghnie, E Genovese, M Grazia Sommaruga, $M$ Aricò, D Locatelli, E Arbustini, S Pezzotta and F Severi, unpublished data). This is also strengthened by the fact that the rapid increase in the inflammatory mass stopped after steroid treatment, because of local vascular and inflammation modifications. In our opinion, there were no effects of the stalk biopsy on these changes as the same MRI pattern was observed in the course of steroid treatment of systemic LCH with unbiopsied hypothalamic granulomatous infiltration (M Maghnie, E Genovese, M Grazia Sommaruga, M A ricò, D Locatelli, E Arbustini, S Pezzotta and F Severi, unpublished data).

To our knowledge, this is the first reported case of an inflammatory tumor-like lesion of the hypothalamus and pituitary stalk associated with abnormal vascularization, suggesting that one pathogenic mechanism leading to the growth of a hypothalamic mass may be a vasculitic lesion in the hypophyseal arterial system causing large local inflammatory reaction and ischemic damage.

Our case demonstrates that evolutive hypothalamicpituitary inflammatory vascular disease should be considered in the differential diagnosis of a hypothalamic mass during childhood. Early definitive diagnosis of an isolated thickened stalk may be difficult, but risky surgery should be undertaken only in selected evolutive cases. On the other hand, it was recently reported that neither early chemotherapy nor radiotherapy of hypothalamic-stalk granulomatosis prevents the usual course of central nervous system-related LCH (23). Close follow-up of a chronic thickened pituitary stalk remains mandatory as is shown here by the results of 7 years of follow-up, including 2 years after treatment began, which also confirmed that the conservative approach was reasonable in our patient. The favorable response to glucocorticoid pulses (after stalk biopsy) underlines the role of steroids in the management of such inflammatory masses and the need to perform ad hoc clinical studies.

\section{Acknowledgements}

We gratefully acknowledge the screening of autoantibodies by Professor Renata Lorini.

\section{References}

1 Mootha SL, Barkovich AJ, Grumbach MM, Edwards ME, Gitelman $\mathrm{SE}$, Kaplan $\mathrm{S}$ et al. Idiopathic hypothalamic diabetes insipidus, pituitary stalk thickening, and the occult intracranial germinoma in children and adolescents. Journal of Clinical Endocrinology and M etabolism 199782 1362-1367.

2 Maghnie M, Villa A, A ricò M, Larizza D, Pezzotta S, Beluffi G et al. Correlation between magnetic resonance imaging of posterior pituitary and neurohypophyseal function in children with diabetes insipidus. Journal of Clinical Endocrinology and M etabolism $199274795-800$.

3 Loh KC, Green A, Dillon Jr W P, Fitzgerald PA, Weidner N \& Tyrrell $J B$. Diabetes insipidus from sarcoidosis confined to the posterior pituitary. European Journal of Endocrinology 1997137 514-519.

4 Maghnie M, A ricò M, Villa A, Genovese E, Beluffi G \& Severi F. MR of the hypothalamic-pituitary axis in Langerhans cell histiocytosis. American Journal of Neuroradiology 199213 1365-1371. 
5 Imura H, Nakao K, Shimatsu A, Ogawa Y, Sando T, Fujisawa I et al. Lymphocytic infundibuloneurohypophysitis as a cause of central diabetes insipidus. New England Journal of M edicine 1993 329 683-689.

6 Pomarède R, Czernichow P, Rappaport R \& Royer P. Le diabète insipide pitressino-sensible de l'enfant. Archives Francais de Pédiatrie 198037 37-44.

7 Greger NG, Kirkland RT, Clayton GW \& Kirkland JL. Central diabetes insipidus in children. American Journal of Diseases and Children $1986140551-554$.

8 Wang LC, Cohen ME \& Duffner PK. Etiologies of central diabetes insipidus in children. Pediatric Neurology 199411 273-277.

9 Fujisawa I, Reinin A, Okumura R, Nakano Y, Shibata T, Hamanaka $D$ et al. Magnetic resonance imaging of neurohypophyseal germinomas. Cancer 19918 1009-1014.

10 Baylis PH \& Gill GV. The investigation of polyuria. Journal of Clinical Endocrinology and M etabolism 198413 295-310.

11 Burgio R, Aricò M, Marconi M, Lanfranchi D, Caselli D \& Ugazio AG. Spontaneous NBT reduction by monocytes as a marker of disease activity in children with histiocytosis. British Journal of Hematology 199074 146-150.

12 Maghnie M, Genovese E, Aricò M, Villa A, Beluffi G, Campani R et al. Evolving pituitary hormone deficiency is associated with pituitary vasculopathy: dynamic MR study in children with hypopituitarism, diabetes insipidus, and Langerhans cell histiocytosis. Radiology 1994193 493-499.

13 Maghnie M, Lorini R, Vitali L, Mastricci N, Carrà AM \& Severi F. Organ- and non-organ-specific auto-antibodies in children with hypopituitarism on growth hormone therapy. European Journal of Pediatrics 1995154 450-453.

14 Ladisch S, Gadner H, A ricò M, Broadbent V, Grois, N, Jacobson A et al. LCH-I: a randomized trial of etoposide vs vinblastine in disseminated Langerhans cell histiocytosis. Medical Pediatric Oncology $199423107-110$
15 Lafitte C, A esch B, Henry-Lebras F, Fetissof F \& Jan M. Granular cell tumor of the pituitary stalk. Journal of Neurosurgery $1994 \mathbf{8 0}$ 1103-1107.

16 Takeuchi J, Kikuchi K, Shibamoto $Y \&$ Fujisawa I. Radiation therapy for juvenile pilocytic astrocytoma of the pituitary stalk. Journal of Neurosurgery $199277139-142$.

17 Hayashi Y, Hamada Y, Oki H \& Yamashita J. Pituitary stalk meningioma: case report. Neuroradiology 199739 351-353.

18 Cosman F, Post KD, Holub DA \& Wordlaw SL. Lymphocytic hypophysitis. Report of three new cases and review of the literature. M edicine 198968 240-246.

19 Thodou E, A sa SL, Kontogeorgos G, Kovacs K, Horvath E \& Ezzat S. Clinical case seminar: Iymphocytic hypophysitis: clinicopathological findings. Journal of Clinical Endocrinology and M etabolism $1995802302-2311$.

20 Ezzat $S \&$ Josse RG Autoimmune hypophysitis. Trends in Endocrinology and M etabolism 19978 74-80.

21 Ahmed SR, Aielo DP, Page R, Hopper K, Towfighi J \& Santen RJ. Necrotizing infundibulo-hypophysitis. A unique syndrome of diabetes insipidus and hypopituitarism. Journal of Clinical Endocrinology and M etabolism 199376 1499-1504.

22 Cemeroglu AP, Blaivas M, Muraszko KM, Robertson PL \& Vàzquez DM. Lymphocytic hypophysitis presenting with diabetes insipidus in a 14-year-old-girl: case report and review of the literature. European Journal of Pediatrics 1997156 684-688.

23 Rosenzweig KE, Arceci RJ \& Tarbell NJ. Diabetes insipidus secondary to Langerhans' cell histiocytosis: is radiation therapy indicated? M edical Pediatric Oncology 199729 36-40.

Received 26 August 1998

Accepted 14 September 1998 